\title{
"La frontera norte tiene tres problemas: tráfico de armas, de drogas y de migrantes"1. Migración irregular y discursos 'securitarios' en Centroamérica: el caso de Costa Rica²
}

Stefanie Kron

Profesora asistente del Departamento de sociología del Instituto de Estudios Latinoamericanos de la Freie Universität Berlin, Alemania. Correo electrónico: skron@zedat.fu-berlin.de

Recibido: septiembre de 2010 / Aceptado: noviembre de 2010

Tomando Costa Rica como caso EJEMPLAR, En EL ENSAYO SE EXPLORA LA TEMATIZACión y el tratamiento institucional de la migración irregular en Centroamérica. Partiendo del paradigma mundial de una "securitización" de la migración irregular (Waever et al., 1993; Weiner, 1995), y aplicando un marco de análisis transnacional y de multiniveles, se preguntará por los repertorios discursivos, los actores y medidas específicos que confluyen en la creación de un nexo migración/(in)seguridad en Centroamérica en general y en Costa Rica en particular. La hipótesis principal es que se está fomentando un nuevo régimen regional de migración y de fronteras que (ya) no proyecta principalmente reforzar los mecanismos de control del territorio nacional, proteger las fronteras y detener las migraciones irregulares. Más bien se trata de establecer, a través de ciertos 'discursos securitarios', una nueva gobernabilidad (Foucault, 1991) de la alta permeabilidad de las fronteras centroamericanas y de los movimientos de los individuos que las cruzan. Usando métodos etnográficos y análisis del discurso (Jäger ,2001), el artículo analiza cómo los 'discursos securitarios' que circulan a nivel mundial y regional se manifiestan, por un lado, en la nueva legislación de migración en Costa Rica, y por otro, en las prácticas de "movilidad transfronteriza" y de control de la misma en la llamada "frontera norte" que separa Costa Rica y Nicaragua.

Palabras clave: migración irregular / fronteras / securitización / gobernabilidad / teoría del régimen 


\section{Introducción}

El informe final de la "Global Commission on International Migration" (GCIM) considera el aumento de la migración irregular como un problema mundial considerable para la soberanía de los Estados nacionales, la seguridad pública y la de las mismas poblaciones de migrantes sin estatus legal (GCIM, 2005, p. 32). ${ }^{3}$

En Norte y Centroamérica, el contexto macro del aumento de la migración irregular es la reorganización (geo-)política y económica de la región, que se inició con el fin de los conflictos armados en Nicaragua (1990), El Salvador (1992) y Guatemala (1996). Uno de los ejes centrales de esta reorganización forma la llamada 'integración económica', es decir, los Tratados de Libre Comercio con los EE.UU. El sociólogo William Robinson reseña el objetivo de estos procesos transnacionales con la integración periférica de los países centroamericanos a la economía global (Robinson, 2003, p. 64).

En este marco, la migración de mano de obra hacia los EE.UU y Canadá ha ido formando parte de los bienes de exportación más importantes de la mayoría de los países del Istmo Centroaméricano; y las remesas monetarias registradas de las poblaciones de migrantes centroamericanos representan entre diez por ciento (Guatemala) y más del 25 por ciento (Honduras) del producto interno bruto (World Bank, 2008, pp. 116, 121).

Así, el número de migrantes del Istmo en Norteamérica se triplicó en la década de los años 90 y se duplicó de nuevo en el año 2000. Es por eso que los EE.UU -desde principios de los años 90- han tratado de regular la inmigración mientras restringen cada vez más las posibilidades para la inmigración legal. No obstante, igual que en el caso de la Unión Europea (UE), estas medidas no han llevado a una reducción de la inmigración total, sino más bien al crecimiento de inmigraciones ilegalizadas.

La dinámica de la ilegalización de una buena parte de la migración internacional va acompañada de la tematización y el tratamiento institucional de la migración irregular como un problema nacional y transnacional de seguridad pública. Este nexo discursivo "migración/(in)seguridad" tiende a criminalizar la migración irregular vinculándola con el crimen organizado y justificando así el aumento de medidas para una "securitización” de la migración irregular. ${ }^{4}$ Esto se manifiesta en nuevos modelos legislativos y figuras jurídicas que priorizan la penalización de la migración irregular y de las redes sociales y comerciales que la facilitan, así como en una creciente presencia de fuerzas de seguridad no sólo en las líneas fronterizas mismas sino también en el interior de los países. ${ }^{5}$

Se puede observar una regionalización o transnacionalización y "multilateralización" de políticas de regulación de la migración, así como un cambio paradigmático del "migration control" hacia el llamado "migration management" (Geiger \& Pécond, 2010). Así, ya hace una década que actores supra nacionales como la "Organización Internacional para las Migraciones" (OIM) consideraron que, con el fin de la guerra fría, las tendencias y los patrones de las migraciones internacionales, al igual que la cantidad y la importancia de los Estados involucrados en la migración internacional, habían cambiado significativamente. La migración internacional, por ende, se había convertido en un "multinational process", 
por lo que "the management of migration can no longer solely be on a unilateral or bilateral basis" (Klekowski von Koppenfels, 2001, p.18). De hecho, además de una casi triplicación de personas migrantes en el mundo, de 75 millones en 1950 a 214 millones en el año 2010 (véase IOM, 2010), ${ }^{6}$ se puede observar una traslación de patrones de migraciones unidireccionales hacia transmigraciones, migraciones circulares y migraciones de tránsito. Por lo mismo la OIM apuntó la necesidad de encontrar:

a regional-level response to changes in international migration trends and a growing recognition that the current international migration system, which was developed in another era with other determents, focal points and completely different balance of power, is no longer appropriate (Klekowski von Koppenfels, 2001, p.18).

La producción académica crítica sobre "securitización” y migración irregular en Europa comparte la idea de que las políticas de regulación de migraciones irregulares de la UE no sólo muestran dimensiones nacionales y binacionales, sino también transnacionales y multilaterales. Partiendo de esta consideración se reconoce cada vez más que las regiones de origen y de tránsito de migrantes irregulares ubicadas más allá de las fronteras externas de la Unión Europea, particularmente Turquía y los Estados de África del Norte, forman espacios importantes de discursos y medidas 'securitarios' (véase i.e. Transit Migration Forschungsgruppe, 2007; Hess \& Kasparek, 2010; Papadopoulos, Stephenson \& Tsianos, 2008).

Si bien es verdad que también la producción académica en Norte y Centroamérica reconoce ampliamente que los países de Canadá a Panamá han ido formando una sola región de integración económica y política, aunque ésta sea fuertemente jerarquizada y dominada por los intereses de los EE.UU., no obstante, la mayoría de los estudios con respecto a la tematización y el tratamiento institucional de la migración irregular en Norte y Centroamérica no aplican una perspectiva regional y transnacional. Más bien, se tiende a conservar lo que se ha llamado "nacionalismo metodológico" (Schiller \& Wimmer, 2003) y por eso tiende a limitarse al análisis de actores, discursos y medidas nacionales o binacionales dentro del espacio económico-político del "Tratado de Libre Comercio de América del Norte" (NAFTA).

Segundo hay que constatar que el Istmo Centroamericano aún es un "punto ciego" con respecto a la producción académica sobre migración y seguridad. Por cierto, autores como Juan Sandoval se han despedido de una concepción nacional-territorial de mecanismos del control de la migración, reconociendo que los EE.UU. aspiran a involucrar a México como "país frontera" (Sandoval, 2005) y de esta manera externalizar el control de la inmigración y de sus fronteras hacia los países al sur del Río Bravo (véase también Castillo, 2003). Sin embargo, hasta el momento la idea de la externalización de fronteras apenas se ha traducido a reflexiones metodológicas o investigaciones empíricas que incluyen a los países de origen y de tránsito de migraciones irregulares del Istmo Centroamericano. Además, los mencionados trabajos aún favorecen la hipótesis de que los discursos y medidas 'securitarios' priorizan medidas legislativas, policiales y militares de control que proyecten cerrar las fronteras y evitar la migración irregular. 
No obstante, una buena parte de las actuales migraciones irregulares que circula en la región Norte y Centroamericana, o se origina en los países del Istmo Centroamericano, transita por los mismos, o se dirige a ellos (Rocha, 2004; 2006). Con respecto a la inmigración de personas de los países centroamericanos a los EE.UU., el US-Census del año 2000 estimó un número de al menos cinco millones de personas viviendo en los EE.UU, de las cuales casi tres millones no tenían residencia legal (US-Census, 2002; véase también GCIM, 2005, p.5; Grund, 2006, p.9). ${ }^{7}$

En cuanto a la migración irregular que nada más circula dentro del Istmo -la llamada migración Sur-Sur- es particularmente Costa Rica el que constituye un país de destino, sobre todo para ciudadanos nicaragüenses. Así, el censo costarricense del año 2000 cuenta 300.000 inmigrantes, de los cuales un 75\% provenía de Nicaragua. Sin embargo se estima hasta un millón de personas de origen nicaragüense viviendo en Costa Rica (véase Villa \& Pizarro, 2002; GCIM, 2005, p.17). ${ }^{8}$ Una nueva población inmigrante importante es de origen colombiano. También en términos generales Costa Rica actualmente muestra, después de México, las dinámicas migratorias más diversificadas de la región, incluyendo también cifras evidentes de emigraciones y migraciones de transito. ${ }^{9}$

Es necesario mencionar también las crecientes migraciones irregulares y transcontinentales que provienen de África, Asia y Europa del Este, y que transitan por el Istmo Centroamericano buscando a llegar a los EE.UU o a Canadá (IOM, 1998, p.7).

La conversión del Istmo Centroamericano en un "espacio de tránsito [y de destino] cada vez más importante" (IOM, 1998, p.7) para las migraciones irregulares se debe a dos hechos: las políticas migratorias norteamericanas cada vez más restrictivas por un lado, y por el otro, las casi inexistentes o poco coherentes políticas de regulación de inmigración y vigilancia de fronteras de los países centroamericanos (véase también IOM, 2002b). De hecho, como apuntan también los geógrafos Pascal Girot y Carlos Granados, la historia postcolonial así como la situación actual de las regiones fronterizas centroamericanas son marcadas por una alta permeabilidad y porosidad, es decir, por la escasez de la presencia de instituciones estatales así como por fuertes relaciones socio-culturales, políticas y económicas de carácter transfronterizo (Girot, 1989; Girot \& Granados, 1997). En otras palabras: hasta hoy en día el control del territorio nacional no se ha intentado o logrado ejecutar en las zonas fronterizas del Istmo.

Es por eso que la OIM -que ya en 1998 consideró "the increasing use of the region as a bridge for irregular migration" como la mayor amenaza para la estabilidad económica y política de toda la región norte y centroamericana- concluyó que no es posible evitar estos movimientos transfronterizos. ${ }^{10}$ La organización también destacó la necesidad de crear políticas migratorias que sean regionalmente coordinadas y coherentes y que, más que evitar las migraciones irregulares, se apuntara a "manejarlas" a través del desenvolvimiento de un "migration management":

En suma, la OIM, ya desde hace muchos años se despidió del nacionalismo metodológico y ha ido analizando Centroamérica como laboratorio transnacionalizado para la creación de nuevas formas regionales de manejar 'problemas' transfronterizos de seguridad como 
el de las más diversas migraciones irregulares y las fronteras muy porosas. La producción académica y crítica al respecto, mientras tanto, es aún muy escasa.

Ante este trasfondo, inspirado por los estudios críticos sobre la transnacionalizacion de las politicas migratorias de la UE, y tomando Costa Rica como caso ejemplar, este ensayo tiene el propósito de explorar la tematización y el tratamiento institucional de la migración irregular en Centroamérica. Partiendo del paradigma mundial de una "securitización" de la migración irregular y aplicando un marco de análisis transnacional de multi-niveles, se preguntará por los repertorios discursivos, los actores y medidas específicos que confluyen en la creación de un nexo migración/(in)seguridad en Costa Rica. ${ }^{11}$ La hipótesis es que, bajo el lema de "migration management", se fomenta un nuevo régimen de movilidad transfronteriza y de fronteras.

En este contexto se recurre al concepto de "régimen" (Keohane, 1982; 1984) porque permite involucrar a actores no-estatales (como ONG) y supra nacionales (como la OIM) al análisis de instituciones y políticas de regulación de la migración, pues estos actores han ido jugando cada vez más un papel propio en la búsqueda de respuestas a los llamados 'desafíos globales', como se considera ser la migración (véase i.e. Brand, 2000). A diferencia de Keohane y su definición funcionalista del concepto de régimen, en el presente ensayo el término "régimen" se entiende siguiendo a Serhat Karakayali y Vassilis Tsianos, como una "regularización de conflictos sociales" y como una productiva "complejidad de dinámicas", cuya "constelación no es dada a priori, sino que consiste en las respuestas a las cuestiones y problemas que resultan de elementos y procesos dinámicos" (Karakayali \& Tsianos, 2007, p.14). Esta definición permite, además, entender la migración como una "relación social", es decir que la "cara subjetiva" y la "fuerza dinámica" que resultan de la misma se tiene que incluir en el análisis del régimen de migración y de fronteras respectivamente (Karakayali \& Tsianos, 2007, p.13).

Volviendo al concepto de "migration management", propuesto por actores internacionales como la OIM, y reajustando la idea del "país frontera" al caso de Centroamérica, propongo que el nuevo régimen de movilidad transfronteriza y de fronteras en Costa Rica proyecta principalmente reforzar los mecanismos de control del territorio nacional y defenderse de las migraciones irregulares. Más bien trata de establecer -a través de ciertos discursos y prácticas 'securitarias' que se centran en lo que llamo discurso "antitrafficking” (Hess \& Tsianos, 2007)- una nueva gobernabilidad (Foucault, 1991) de la alta permeabilidad de las fronteras y de los movimientos de los individuos que las cruzan. Esta nueva forma de gobernabilidad tiende a subjetivar el individuo de movilidad transfronteriza ("Grenzgänger") como un "potencial portador de riesgos" (Braig \& Baur, 2005, p.183).

Por lo tanto, en la primera parte del presente texto se esbozan los actores y discursos transnacionales y regionales relevantes para el análisis del nexo migración/(in)seguridad a nivel nacional en Costa Rica. En la segunda parte se analiza cómo los 'discursos securitarios' se manifiestan en la nueva legislación de migración en Costa Rica y cómo se produce y reproduce el nuevo régimen de movilidad transfronteriza en la frontera Costa Rica - Nicaragua, la llamada "frontera norte". A esta frontera la conceptualizo como un espacio social conflictivo y en negociación, en donde confluyen procesos locales, nacionales 
y transnacionales que constantemente crean nuevas dinámicas entre prácticas de movilidad transfronteriza y de regulación de movilidad (véase Bernecker, 2005). ${ }^{12}$

\section{El discurso antitrafficking: re-codificando la migración irregular a nivel mundial y regional}

En noviembre de 1989, pocos días después de la caída del muro de Berlín, entró en vigor una extensa reforma del "International Committee for European Migration" (ICEM), una pequeña agencia dominada por los EE.UU. que hasta ese momento organizaba la emigración de europeos a las Américas y Australia. Ésta se renombró llamándose desde entonces "International Organization for Migration" (IOM). ${ }^{13} \mathrm{En}$ los años siguientes la OIM aumentó la cantidad de sus Estados miembros de 36 a 86, cambó sus competencias hacia la promoción y organización de la "movilidad ordenada" de todo tipo de migrantes internacionales, y se convirtió en la organización supra nacional más importante del mundo con respecto a discursos, prácticas y políticas en el campo del "management" de la migración internacional. Sin embargo, desde principios de los años 90 la problematización y el tratamiento de la migración irregular se convirtieron en el área de incidencia más importante de la OIM. En este contexto, la organización empezó a poner énfasis en la recolección y distribución de información acerca de lo que se ha nombrado, desde entonces, "smuggling of migrants" (en castellano: "tráfico ilícito de migrantes") y "trafficking of humans" ("trata de personas"). Luego, a partir de mediados de los 90, la OIM impulsó la creación de nuevas instituciones, modelos legislativos y figuras jurídicas que permitieran criminalizar y penalizar estas formas del crimen organizado (véase Georgi, 2010, p.146).

A continuación quisiera realzar dos de las instituciones claves que han contribuido a crear un lenguaje común a nivel mundial con respecto a la criminalización del "smuggling" y del "trafficking". Se trata primero de los denominados "Protocolos de Palermo" adoptados por las Naciones Unidas en el año 2000 y segundo, de los "Procesos Regionales de Consulta sobre Migración” (RCP).

\subsection{Los Protocolos de Palermo}

Los "Protocolos de Palermo" conforman la "Convención contra el Crimen Organizado Transnacional". Consisten en el "Protocol to Prevent, Suppress and Punish Trafficking in Persons Especially Women and Children”, que entró en vigor en 2003 (UN Convention Transnational Crime, Trafficking, Annex II, 2000: Art. 3, p. 2); y en el "Protocol against the Smuggling of Migrants by Land, Sea and Air”, que entró en vigor en 2004 (UN Convention Transnacional Crime, Smuggling, Annex III, 2000: Art. 3, pp. 2-3).

Los Protocolos definen smuggling y trafficking de la siguiente manera:

'Smuggling of migrants' shall mean the procurement, in order to obtain, directly or indirectly, a financial or other material benefit, of the illegal entry of a person into a State Party of which the person is not a national or a permanent resident (UN Convention Transnational Crime, Smuggling, Annex III, 2000: Art. 3, S. 2). 
'Trafficking in persons' shall mean the recruitment, transportation, transfer, harboring or receipt of persons, by means of the threat or use of force or other forms of coercion, of abduction, of fraud, of deception, of the abuse of power or of a position of vulnerability or of the giving or receiving of payments or benefits to achieve the consent of a person having control over another person, for the purpose of exploitation. Exploitation shall include, at a minimum, the exploitation of the prostitution of others or other forms of sexual exploitation, forced labor or services, slavery or practices similar to slavery, servitude or the removal of organs (UN Convention Transnational Crime, Trafficking, Annex II, 2000: Art. 3, S. 2).

\subsection{Los Procesos Regionales de Consulta}

A partir de 1995 la OIM promovió y coordinó la formación de por lo menos 15 denominados "Procesos Regionales de Consulta sobre Migración" (RCP) en casi todo el mundo (véase Klein-Solomon, 2005). ${ }^{13}$ Los RCP son foros informales, flexibles y abiertos. Operan en forma de red y reúnen a actores nacionales y supra nacionales con el fin de debatir asuntos migratorios a nivel regional, de crear alianzas que finalmente deberían resultar en nuevos acuerdos bilaterales y multilaterales, y de contribuir al establecimiento de un nuevo régimen de migración internacional (véase Hansen, 2010; Klekowski von Koppenfels, 2001; Thouez $\&$ Channac, 2005).

En este contexto, el tema más importante de la mayoría de los RCP es la problematización de la migración irregular como una potencial forma de crimen organizado transfronterizo mientras la vincula con "smuggling" y "trafficking”. Así, la migración irregular reaparece como problema de seguridad pública transnacional que habría que "manejar" o combatir a los niveles multilateral y transnacional. Amanda Klekowski von Koppenfels, quien elaboró un informe de evaluación sobre los RCP para la OIM, escribe al respecto:

[T] here is now more irregular migration as well as smuggling and trafficking of migrants. Trafficking today is again and again referred to as a growing and changing problem. It appears that at least some trafficking and smuggling is controlled by organized crime, meaning that traffickers/smugglers have a wide network upon which they can draw to outwit or avoid law enforcement authorities. While some of the organized trafficking/smuggling groups appear to be very small operations, others are international in scope and are thus able to function effectively across borders [...]. Logically, then, governments could be more effective in combating smuggling/trafficking were they also to work together on this issue (Klekowski von Koppenfels, 2001, p.17).

Es importante anotar que esta vinculación discursiva de la migración irregular con "smuggling" y "trafficking" no sólo muestra elementos criminalizantes sino también victimizantes, los cuales se activan a través del discurso de derechos humanos: "Combating trafficking is, indeed, [...] a topic, more than others, that appears to concern sending and receiving countries equally, largely because of the grave human rights abuses inherent in trafficking and the potential for such abuses in smuggling“" (Klekowski von Koppenfels, 2001, p.31). 
Las citas evidencian la construcción discursiva de un actor criminal, amenazante, extralegal y 'privado' -el "smuggler" o "trafficker" - que amenaza la seguridad pública de los Estados afectados por la migración irregular y viola sistemáticamente los derechos humanos de migrantes irregulares. Al mismo tiempo se construye al migrante irregular como una víctima de estas formas del crimen organizado. Mientras tanto, debates sobre poblaciones de migrantes irregulares como portadores de derechos laborales, de derechos a la salud o a la educación etc. y de cómo los Estados involucrados en la migración internacional podrían garantizar estos derechos, no forma parte de los discursos dominantes de los RCP.

El informe actual de evaluación de los RCP considera que se logró que muchos Estados miembros adoptaran políticas respecto a este asunto; que en casi todos los países que forman parte de uno o más RCP la criminalización del "trafficking" forma una práctica común y que algunos Estados han usado el modelo legislativo de los "Protocolos de Palermo" para diseñar legislaciones nacionales al respecto (Hansen, 2010, pp.29-30).

Así, los "Protocolos de Palermo" y los discursos de los RCP han contribuido a reducir las múltiples facetas y prácticas de la migración irregular a los conceptos anglo-sajones de "smuggling/trafficking" y de esta manera han logrado criminalizar o victimizar los más diversos actores involucrados en la migración irregular.

Sabine Hess y Vassilis Tsianos denominan esta reformulación de la migración irregular "discurso antitrafficking", y consideran que es un "discurso securitario" dominante que "recodifica" la migración irregular en términos de crimen organizado (Hess y Tsianos, 2007, p.29). Según los mismos autores, el "discurso antitrafficking" también tiende a objetivar a las múltiples subjetividades de la migración irregular, es decir, a los migrantes mismos, pero también a los transportistas, comerciantes, dueños de hospedajes, etc. que forman las redes facilitadoras, a sólo dos figuras discursivas binarias: "delincuente" (smuggler/trafficker) y "víctima" (migrante irregular) (véase también Papadopolous et.al, 2008). De esta manera, el debate sobre la migración irregular se ha ido desvinculando ampliamente de cualquier contextualización social y de la cuestión de derechos básicos en la migración. En otras palabras, se han ido invisibilizando los conflictos y desigualdades sociales que condicionan la migración irregular. Además, el "discurso antitrafficking" tiende a diluir las importantes diferencias, definidas por los "Protocolos de Palermo", entre "smuggling" (facilitación comercial de la migración irregular) y "trafficking" (comercio con seres humanos con el fin de explotación sexual o laboral). ${ }^{14}$

\subsection{La Conferencia Regional sobre Migración en Norte y Centroamérica}

A continuación se precisarán estas consideraciones para el nivel de la "Conferencia Regional de Migración / Regional Conference on Migration” (CRM/RCM), la cual constituye el RCP para Norte y Centroamérica y es también conocida como "Proceso Puebla". La CRM/ RCM forma parte de los primeros RCP que se crearon (1996). Al mismo tiempo, es uno de los RCP más consolidados. Es por eso que tiene carácter de modelo para otros RCP (véase Hansen, 2010; IOM, 2002b; Klekowski von Koppenfels, 2001; RCM/CRM, 2004 y 2006; Thouez \& Channac, 2005). 
Con respecto al organigrama, al financiamiento y a los actores principales de la CRM/RCM se puede resumir que los financieros más importantes son los EE.UU y Canadá. Entre los países participantes con voto figuran -aparte de los principales financieros mencionadosMéxico, Belice, Guatemala, Honduras, El Salvador, Nicaragua, Costa Rica, Panamá y la República Dominicana. Argentina, Ecuador, Colombia, Jamaica y Perú participan como países observadores. Entre las organizaciones cooperantes figuran actores supra nacionales como la OIM pero también el "Alto Comisionado de las Naciones Unidas para Refugiados" (ACNUR), el "Programa de Naciones Unidas para el Desarrollo" (PNUD), la "Comisión Económica para América Latina" (CEPAL), el United Nations Children's Fund" (UNICEF) y el "United Nations Population Fund" (UNFPA), entre otros, así como instituciones regionales como la "Comisión Centroamericana de Directores de Migración" (OCAM) y el "Sistema de Integración Centroamericana" (SICA). La CRM/RCM es, además, uno de los pocos RCP que incluye, a nivel de observadores, a una red de ONG con el nombre "Red Regional de Organizaciones Civiles para las Migraciones" (RROCM). También existe una unidad especial, la "Red Regional para combatir el Tráfico y la Trata de Personas". La $\mathrm{CRM} / \mathrm{RCM}$ celebra reuniones anuales donde participan los/las viceministros/as del Interior y de Asuntos Exteriores de los países participantes, así como representantes de los Estados y de las organizaciones observadores. La presidencia rota entre los países participantes. La CRM/RCM también mantiene una secretaría técnica, manejada por la oficina regional de la OIM para México y Centroamérica, ubicada en San José, Costa Rica (véase RCM, 2006; Hansen, 2010).

Dominada por el interés de los EE.UU. de involucrar a México y los países del Istmo Centroamericano como "países fronteras" (Sandoval, 2005) los debates y acciones políticas de la CRM/RCM son, aún más explícitamente que en el caso de otros RCP, caracterizados por un discurso de una candente amenaza de las migraciones irregulares para la seguridad ciudadana y la estabilidad política y económica en toda la región:

The Puebla Process emerged due to the concern over irregular migration affecting the entire region. [...] On the basis of this initial framework, the Puebla Process was initiated with the firm intention of reducing irregular migration in the North and Central American region (Klekowski von Koppenfels, 2001, p.34).

Desde su creación en 1996 hasta el año 2000 se trabajó, por ende, en la elaboración y aplicación de un "Plan de Acción" que evidencia la predominancia del nexo migración/(in) seguridad. Así, entre los objetivos principales contaban:

Armonizar las categorías migratorias y los requisitos para la inmigración en todos los países participantes. ${ }^{15}$

Cooperar en combatir el tráfico y la trata de personas a través del desenvolvimiento de un sistema regional de información mejorado y luego por la creación de leyes nacionales contra el tráfico y la trata y para la protección de las víctimas.

- La cooperación técnica para mejorar la eficiencia del control de fronteras y de procesamiento penal del tráfico y de la trata.

- La cooperación en la expulsión y repatriación de migrantes extra regionales (Klekowski von Koppenels, 2001, pp.35-36). 
En este contexto vale la pena anotar que en el marco de la CRM/RCM la traducción del concepto "smuggling of migrants" al castellano es "tráfico ilícito de personas", lo que es conocido popularmente como "coyotaje" o "coyoteada" en el vocabulario de la región centroamericana. El concepto de "trafficking of humans", por su parte, se tradujo a "trata de personas".

La reunión anual de la CRM/RCM del año 2000 llegó a la conclusión de que la mayoría de los objetivos formulados en el "Plan de Acción" fueron conseguidos, o por lo menos su realización fue impulsada. Luego se elaboró un llamado "Documento de Visión" que pone énfasis a la modernización del "migration and border management" y se acordó concederle a la CRM/RCM una base más operativa (véase Klekowski von Koppenels, 2001, p.34). Particularmente desde el 11 de septiembre de 2001 este proyecto está -aún mucho más que el "Plan de Acción" de 1997- marcado por el nexo migración/(in)seguridad que se centra en el discurso antitrafficking. La siguiente cita, tomada del informe final del "Workshop on Migration Management and Improving Inter-State Cooperation", realizado por el "Migration and Policy Research Programme” (MPRP) de la OIM para los Estados miembros de la CRM/ CRM, evidencia esto con toda claridad:

Participants had no difficulty identifying the major policy challenges that they face or are likely to face in the near future. At or near the top of all agendas was a preoccupation with the migration - security linkage. Clearly, the concept of facilitation of movement is acceptable to governments only if security issues are taken care of [...]. The combat against migrant smuggling and trafficking was also of concern to all delegations, given especially, it's devastating impact on the victims of smugglers and traffickers. At the point of departure the latter charge extortionate fees; during the clandestine border crossing they manifest little or no regard for the physical safety or even the lives of their clients; and at the other end they leave them vulnerable to exploitation and abuse. Delegations saw the need, therefore, for both measures to combat smugglers and traffickers, and to protect their victims. Closely related to the issue of smuggling and trafficking is the broader phenomenon of irregular migration, which calls, not for a single policy response, but for a series of across-the-board mutually supportive measures. Participants emphasized that ideally these should include 'upstream' (or preventive) action such as information campaigns or population stabilisation programmes, as well as 'downstream' (control, compliance and return of unauthorized arrivals) action (IOM, 2002, p.2; acentuaciones en el original).

Los informes de Klekowski von Koppenfels (2001) y de Hansen (2010) consideran la CRM/ RCM como uno de los RCP más exitosos en cuanto a la realización de sus objetivos: la cooperación regional en combatir el tráfico de migrantes y la trata de personas, la intensificación de los mecanismos de vigilancia de fronteras y la elaboración de nuevos mecanismos para la regulación de la migración en general.

\section{3. 'Securitizando’ la migración y las fronteras: el caso de Costa Rica}

Este capítulo está dedicado al análisis de cómo el discurso antitrafficking se manifiesta en la nueva ley de migración en Costa Rica y cómo contribuye al establecimiento de un nuevo régimen y una nueva gobernabilidad de la movilidad transfronteriza y de fronteras en ese país. 


\subsection{La nueva ley de migración: el marco normativo del nexo migración/(in)seguridad}

En marzo de 2010 entró en vigor una nueva ley de migración en Costa Rica que se ha ido discutiendo en varias versiones desde principios de los años 2000 (Huhn, 2005, p.86; Sandoval, 2007, pp.xiv/xv). Se trata de una reforma de la "Ley General de Migración y Extranjería, No. 8764" que fue aprobada en 2006 y que reemplazó la "Ley General de Migración y Extranjería, No. 7033” de 1986. Muchos académicos y juristas pro-migrantes, así como representantes de ONG opinan que la actual reforma legislativa toma bastante en cuenta los derechos humanos y ciudadanos de las poblaciones migrantes, mientras que la versión de 2006 reducía aún más la migración a un asunto de "seguridad pública" (Sandoval, 2007, p.xv). Sin embargo, la "Ley General de Migración y Extranjería, No. 8764", sea la versión de 2006 o de 2010, define un marco normativo completamente nuevo y forma el fundamento legislativo-institucional para la criminalización y penalización de la migración irregular y de las redes que la facilitan. ${ }^{16}$

Para tal fin, cuenta primero que la nueva legislación prevé la reorganización de la policía de migración con el fin de aumentar su área de incidencia y sus competencias hacia propias investigaciones judiciales "en el campo migratorio, contra la corrupción y la criminalidad organizada transnacional" y "sobre los delitos de tráfico y trata de personas" (Ley General de Migración y Extranjería, título 4, artículo 18; 2 y 26). Anteriormente, sólo el "Organismo de Investigación Judicial de Costa Rica" (OIJ) tenía la autorización de realizar investigaciones judiciales.

Segundo, aparecen novedosas figuras jurídicas en la nueva ley de migración que la legislación anterior no incluía. Se trata por un lado del delito del "tráfico ilícito de personas" que se define de la manera siguiente:

Se impondrá pena de prisión de dos a seis años a quien conduzca o transporte a personas, para su ingreso al país o su egreso de él, por lugares no habilitados por las autoridades migratorias competentes, evadiendo los controles migratorios establecidos o utilizando datos o documentos falsos o alterados. La misma pena se impondrá a quien, en cualquier forma, promueva, prometa o facilite la obtención de tales documentos falsos 0 alterados y a quien, con la finalidad de promover el tráfico ilícito de migrantes, aloje, oculte o encubra a personas extranjeras que ingresen 0 permanezcan ilegalmente en el país (Ley General de Migración y Extranjería 8764, título 15 , artículo 249). ${ }^{17}$

La figura jurídica del "tráfico ilícito de personas" permite, entonces, criminalizar y penalizar duramente las múltiples y diversas prácticas del coyotaje y del apoyo comercial y no comercial a migrantes irregulares. Entre estas prácticas cuentan el alojamiento y la alimentación, la mordida para las autoridades públicas del control fronterizo, la facilitación del tránsito diario de personas de lugares cercanos a la frontera a través de los puntos ciegos que se realiza a pie, en taxis, vehículos particulares, motos, camiones y botes, así como formas más organizadas del coyotaje a través de varias fronteras, países y continentes. 
En este contexto vale la pena mencionar que el año pasado también se reformó el código penal que ahora por primera vez incluye explícitamente el delito de "trata de personas", es decir el trafficking, que se define así:

Será sancionado con pena de prisión de seis a diez años, quien promueva, facilite o favorezca la entrada o salida del país, o el desplazamiento dentro del territorio nacional, de personas de cualquier sexo para realizar uno o varios actos de prostitución o someterlas a explotación, servidumbre sexual o laboral, esclavitud, trabajos o servicios forzados, matrimonio servil, mendicidad, extracción ilícita de órganos o adopción irregular (Código Penal de Costa Rica, artículo 172).

Por otro lado, la nueva legislación de migración inventó la categoría migratoria de "víctima de trata de personas". Es una categoría migratoria especial y temporal. La persona considerada "víctima de trata" goza de ciertos derechos y no puede ser deportada con el fin de "regular situaciones migratorias que, por su naturaleza, requieran un tratamiento diferente de las categorías migratorias" (Ley General de Migración y Extranjería 8764, título 6 , capítulo 4, artículos 93 y 94; 19).

Para contrastar la ley con la realidad estadística quisiera anotar que la base de datos respecto a la "trata de personas" es muy escasa. No se encuentran en ningún lado cifras exactas de este hecho en Costa Rica. La coordinadora de la "Unidad contra el tráfico ilícito y la trata de personas" de la oficina regional de la OIM para México y Centroamérica en San José habla de 11 casos denunciados y de un caso procesado en los últimos tres años (entrevista, 13 de mayo de 2010).

Se puede resumir que la nueva ley de migración en Costa Rica convirtió en figuras jurídicas las dos figuras binarias discursivas del "discurso antitrafficking": el "traficante/tratante" por un lado y la "víctima" por el otro. En el contexto de este nuevo marco jurídico, la categoría de la víctima es el único estatus legal que los migrantes irregulares pueden obtener sin ser penalizados o deportados y que les garantice ciertos derechos sociales y de protección. Pero son derechos limitados al período en que se regule la "situación migratoria". Es un estatus, entonces, parecido al estatus del refugiado.

Además, como confirma la coordinadora de la "Unidad contra el tráfico ilícito y la trata de personas" de la OIM en San José, la nueva ley de migración no sólo está fuertemente influida por el modelo legislativo de los Protocolos de Palermo y por el "documento visión" de la CRM, sino que la misma OIM participó activamente, como agencia de consultoría legal y política, en el diseño de la ley así como en la reforma del código penal (entrevista, 13 de mayo de 2010).

\section{2. "Discursos securitarios" y la nueva gobernabilidad de la frontera norte de Costa Rica}

En este último apartado analizaré cómo el "discurso antitrafficking" influye en los actores y las prácticas de la movilidad transfronteriza y de la regulación de la misma, fomentando de esta manera una nueva gobernabilidad de la frontera norte de Costa Rica. 
Al igual que otras fronteras de la región centroamericana, la frontera que separa Costa Rica de Nicaragua es una frontera altamente porosa y permeable. En el trayecto de los 300 kilómetros de la línea fronteriza existen solamente dos puestos fronterizos habilitados (puestos de migración): primero Peñas Blancas (provincia Guanacaste), que es un paso terrestre, y segundo Los Chiles (provincia Alajuela), que es un paso por río. En cambio, hay muchísimos punto ciegos, es decir, pasos de frontera no autorizados: por lo menos siete carreteras o sendas transfronterizas y una cantidad incontable de cruces por ríos.

Como ya se mencionó en un principio, históricamente la presencia de instituciones estatales en esta zona siempre ha sido débil, ya que hasta la revolución sandinista en el año 1979, ni el Estado nicaragüense ni el Estado costarricense intentaban ejercer control del territorio nacional en el sentido de una marcación de la soberanía nacional. Más bien, las relaciones de poder estaban marcadas por actores privados y actividades transfronterizas. En el caso de la provincia de Guanacaste, este fenómeno se inició en el siglo XVII con la fundación de la ciudad de Liberia por familias de ganaderos provenientes de la ciudad de Rivas en Nicaragua. En la provincia de Alajuela, mientras tanto, las dinámicas transfronterizas se impulsaron a finales del siglo XIX con los cimientos de la ciudad de Upala por comerciantes nicaragüenses (véase Medina-Nicolas, 2004; Núñez \& Marín, 2009). A todo esto se suma que la zona fronteriza formaba históricamente una brecha para actividades y proyectos de actores transnacionales que estuvieron interesados en construir un canal interoceánico en esta zona, que también debería beneficiar a los gobiernos de los dos países (Girot, 1989; Girot \& Granados, 1997; Huhn, 2005).

\subsection{Actores y prácticas de la movilidad transfronteriza}

Es por todo esto que, hasta hoy en día, la cotidianidad de la vida y de la sobrevivencia en cantones fronterizos como La Cruz -donde queda el puesto de migración de Peñas Blancasy Upala está marcada por cruces no autorizados de la frontera. La población que vive a lo largo de la línea fronteriza entre Peñas Blancas y Upala y en las comunidades respectivas del lado nicaragüense usa una gran variedad de puntos ciegos y formas de transporte para cruzar la línea. Los motivos son variados: trabajo, comercio, visitar a familiares, obtener servicios sociales básicos, etc. Este es particularmente el caso en el cantón de Upala y en las comunidades nicaragüenses vecinas, lugares que no tienen ningún puesto de migración cerca.

Una selección representativa de esta gran variedad de actores y prácticas de la movilidad transfronteriza y del diario cruce fronterizo irregular se presentará a continuación. Son casos ilustrativos de grupos paradigmáticos como comerciantes, pobladores de comunidades fronterizas al lado nicaragüense, profesionales de ONG, trabajadores transfronterizos, transportistas, migrantes irregulares de tránsito, oficiales públicos y coyotes profesionales.

\subsubsection{Los comerciantes}

Las diferencias económicas enormes entre Costa Rica y Nicaragua en cuanto a los costos de producción, los salarios y precios de venta, condicionan que el tráfico con productos nicaragüenses constituya una actividad económica muy atractiva para comerciantes nicaragüenses y costarricenses en la zona fronteriza del lado costarricense. 
Un ejemplo entre muchos es el tráfico transfronterizo de sandalias de piel. En el mercado de la ciudad nicaragüense de Masaya funciona una gran cantidad de pequeños talleres de zapatos. Según varios artesanos entrevistados, las sandalias se producen a un precio de entre tres y cuatro dólares por par (entrevista con artesanos de Masaya, 30 de agosto de 2010). Los denominados "intermediarios" llevan esta mercancía a centros comerciales costarricenses en zonas fronterizas, como Upala, donde venden las sandalias a dueños de tiendas de "variedades" a un precio de entre cinco y seis dólares. Los comerciantes de Upala, por su lado, venden las sandalias a un precio de entre ocho a diez dólares (entrevista a matrimonio Rivera, Upala, 27 de abril de 2010).

Entre estos comerciantes de Upala figura el matrimonio Rivera ${ }^{18}$ que proviene de la Isla de Ometepe en Nicaragua. Los dos poseen y manejan una pequeña tienda de ropa, zapatos y suvenires enfrente de la terminal de buses en el centro de la ciudad. Los zapatos y las sandalias los compran a través de un "intermediario" en Masaya. La señora Rivera afirma viajar a Nicaragua frecuentemente -por lo menos unas seis veces al año- para visitar a sus familiares o para reunirse con los productores e intermediarios de su mercancía en Masaya.

Los Rivera tienen ciudadanía nicaragüense pero disponen de cédulas de residencia costarricenses. Sin embargo, aunque ya tienen 17 años viviendo en Upala, no disponen de pasaporte ni costarricense ni nicaragüense. Esta situación les permite salir de Costa Rica legalmente, i.e. por Peñas Blancas, pero no reingresar documentados, ya que para esto se necesitaría un pasaporte. Es por eso que la pareja suele salir legalmente pasando por Peñas Blancas y reingresar clandestinamente por un punto ciego cerca del pueblo fronterizo de Santa Cecilia, contando con una posible mordida de "varios rojos", billetes de mil colones (ca. dos dólares) para los funcionarios de la policía nacional (Fuerza Pública), quienes sólo de vez en cuando salen a patrullar ese lugar (entrevista a matrimonio Rivera, Upala, 27 de abril de 2010).

\subsubsection{Los pobladores de las comunidades rurales al lado nicaragüense}

A pocos kilómetros de Upala se encuentran unas ocho comunidades fronterizas y rurales que están ubicadas en la orilla del lago de Nicaragua y que, formalmente, pertenecen a la provincia nicaragüense de San Carlos. Sin embargo, éstas no tienen acceso directo a una carretera que les llevaría en menos de un día de viaje a un poblado en Nicaragua que dispusiera de servicios sociales básicos como hospitales, escuelas, etc. Pero las comunidades están conectadas con la ciudad de Upala por una carretera de terracería que pasa por la comunidad de México, ubicada en la mera línea fronteriza al lado costarricense. Es por eso que los pobladores de las comunidades fronterizas nicaragüenses suelen viajar indocumentados a Upala, cruzando la frontera en el punto ciego de la comunidad de México, con el fin de ir a la escuela, al hospital etc. (véase i.e. video www.canal2.com.nica: "Educación sin fronteras").

\subsubsection{Los profesionales de ONG}

La coordinadora de la sede upaleña de una ONG costarricense de apoyo a migrantes trabaja en proyectos de salud, de educación y de capacitación de mujeres en las mencionadas 
comunidades vecinas de Upala al lado nicaragüense. La mujer de 23 años tiene ciudadanía y pasaporte nicaragüense así como cédula de residencia en Costa Rica porque está casada con un ciudadano costarricense y tiene un hijo con él que nació en Costa Rica. La entrevistada transita la frontera varias veces a la semana para visitar a sus contrapartes de proyectos comunitarios al lado nicaragüense. Nunca cruza la frontera 'legalmente' por uno de los dos puestos fronterizos habilitados, ya que tanto Peñas Blancas como Los Chiles quedan a más de 50 kilómetros de distancia, mientras que las comunidades nicaragüenses donde ella trabaja quedan a pocos kilómetros de Upala. La entrevistada, por ende, suele cruzar la frontera por el mismo punto ciego de la comunidad de México que sus vecinos nicaragüenses (entrevista a coordinadora ONG, Upala, 27 de abril de 2010).

\subsubsection{Los trabajadores transfronterizos}

Los trabajadores agrícolas temporales de Nicaragua forman otro importante grupo de actores de frontera. Particularmente durante las épocas de cosecha en las grandes plantaciones de naranja, cana, y piña -que en los últimos 10 a 15 años se han ido extendiendo a los dos lados de la línea fronteriza- miles de trabajadores de todas partes de Nicaragua cruzan la frontera -con apoyo de coyotes o a cuenta propia- irregularmente por una gran variedad de puntos ciegos a lo largo de la línea fronteriza entre Peñas Blancas y Los Chiles. ${ }^{19}$

\subsubsection{Los transportistas}

Los movimientos transfronterizos y diarios que se han esbozado arriba se realizan a pie, en bote, bicicleta, moto, bus (hasta la línea fronteriza), taxi formal ("taxi rojo") o informal ("taxi pirata"). Son particularmente los taxistas quienes facilitan la movilidad transfronteriza: circulan en todas partes de la zona fronteriza, llevan a sus clientes hacia la línea fronteriza y algunos, principalmente los choferes de taxis piratas, incluso cruzan la línea. En Upala, particularmente, hay una buena cantidad de choferes de taxis piratas que (sobre-)viven del transporte a la frontera 0 a través de la línea fronteriza. ${ }^{20}$

\subsubsection{Los migrantes irregulares de tránsito}

Las migraciones irregulares de carácter transnacional -o incluso transcontinental- y de tránsito, que en su mayoría atraviesan Costa Rica y otros países centroamericanos con el fin de llegar a los EE.UU., suelen cruzar la frontera por el mismo puesto de migración de Peñas Blancas o cerca del mismo, ya que la carretera Interamericana pasa por este lugar, lo que es esencial para poder avanzar en el camino a buen paso.

Un día de abril de 2010, por casualidad, fuimos testigos de la detención de un grupo de seis personas -supuestamente de ciudadanía somalí- en el puesto de migración de Peñas Blancas. Según el funcionario de la Fuerza Pública que realizó la detención, el grupo había ingresado dos semanas antes por el puesto de migración de Paso Canoas en la frontera sur del país. En ese lugar, comenta el mismo informante, el grupo de africanos se entregó a las autoridades pidiendo asilo político. Luego se los trasladó a San José con el fin de tramitar sus solicitudes. Una vez en San José, el grupo desapareció y reapareció días después en Peñas Blancas, donde fue detenido mientras intentaba pasar cerca de las instalaciones de 
Migración (entrevista a funcionario de la Fuerza Pública, Peñas Blancas, 24 de abril de 2010).

Según confirma un agente de la Fuerzas Pública del pueblo fronterizo de Paso Canoas, donde se encuentra el puesto de Migración para cruzar la frontera con Panamá, la práctica de pedir asilo político al ingresar a Costa Rica por la frontera sur y luego salir irregularmente del país, se ha convertido en una práctica común entre los migrantes de tránsito, particularmente para el creciente número de personas provenientes de Somalia y Etiopía, que aspiran a llegar a los EE.UU. El mismo informante también comentó que las detenciones de migrantes de tránsito con origen africano son muy raras pues la mayoría son "víctimas" de redes de traficantes y tratantes de personas bien organizadas a nivel transcontinental (entrevista a funcionario de la Fuerza Pública, Paso Canoas, 26 de julio de 2010)

\subsubsection{Los funcionarios públicos y los coyotes}

Hasta el momento, aparte del caso excepcional de la detención de los Somalíes en Peñas Blancas, todas las demás prácticas de cruces irregulares de frontera se han podido realizar gracias a pases de frontera no controlados o a "acuerdos informales" con los funcionarios públicos de control de frontera, sea esto a base de tolerancia $o$ a base de mordidas a una escala relativamente pequeña. Particularmente, el atractivo del paso de frontera formal de Peñas Blancas para los cruces irregulares de migrantes de tránsito significa que los mismos funcionarios públicos, es decir, los agentes de la Fuerza Pública, de la policía de migración y de la aduana, forman parte de los actores principales que facilitan los pases indocumentados -cerrando los ojos, cobrando dinero o pidiendo "favores sexuales". Además, varios grupos de coyotes tienen su "oficina" en el salón de billar de "Pedro's bar", ubicado frente a las instalaciones de la aduana costarricense (véase i.e. Villalobos, Chamizo \& Piedra, 2008).

\section{Actores y prácticas de control}

Resultaría, pues, dificilísimo controlar la línea fronteriza territorialmente y evitar los cruces irregulares. Pero como se mencionó en un principio y con respecto a las fronteras centroamericanas, el control del territorio nacional y la supresión de los movimientos transfronterizos e irregulares no forman parte de las prioridades del concepto regional para la modernización del "migration and border management" en el Istmo Centroamericano.

Más bien, el objetivo central consiste -también en el caso de la frontera norte de Costa Ricaen fomentar una nueva gobernabilidad de los individuos móviles que circulan de manera irregular en la zona fronteriza. Para ello, igual que a nivel global y regional, varios actores estatales y públicos, supra nacionales e internacionales, y de la sociedad civil, contribuyen, como se precisará a continuación, a la creación del nexo migración/(in)seguridad centrado en el "discurso antitrafficking" tanto a nivel del discurso sobre migración como a nivel de la materialidad. 


\subsection{Agentes de las fuerzas de seguridad}

Los actores de las fuerzas de seguridad costarricenses, aunque por una lado facilitan los cruces irregulares, al mismo tiempo forman parte de los actores principales del "migration and border managment" mientras recurren al "discurso antitrafficking" diluyéndolo con discursos de tráfico de armas y drogas: así, de los más de 20 funcionarios de la policía entrevistados, todos difunden similares representaciones de la frontera norte. Éstas giran alrededor de la imagen de una "bodega" cada vez más dominada por carteles transnacionales del crimen organizado -particularmente de México y Colombia- y por la caótica circulación de bienes no autorizados y de personas "ilegales", de las cuales se supone que en su mayoría son "víctimas" de tráfico ilícito de migrantes y de trata de personas. El subdirector de la Fuerza Pública de la región 5 ("Zona Norte”), que incluye partes de las provincias de Guanacaste y Alajuela, resume estas supuestas amenazas para la seguridad pública y los consiguientes desafíos más importantes para la Fuerza Pública de la zona fronteriza norte de la manera siguiente: "La frontera norte tiene tres problemas: tráfico de armas, de drogas y de migrantes” (entrevista a subdirector de la Fuerza Pública, región 5, 09 de abril de 2010).

A nivel de la materialidad, estos discursos criminalizantes/victimizantes se traducen en una presencia policial reforzada, no en la misma línea fronteriza, sino más bien en los puntos estratégicos a lo largo de las rutas principales que llevan a la misma. Según confirma el mismo informante policial, se puede hablar, entonces, de un radio de unos 100 kilómetros que se define como "espacio fronterizo" y donde la presencia policial ya se ha intensificado o será intensificada. Esta presencia policial reforzada consiste, además, en una combinación de varias formas de policía, es decir Fuerza Pública, Policía Control de Drogas (PCD) y Policía de Migración (entrevista a subdirector de la Fuerza Pública, región 5, 09 de abril de 2010).

\subsection{Actores internacionales y de la sociedad civil}

Desde hace unos cinco años las sedes regionales de actores supra nacionales einternacionales como la OIM, el ACNUR y la UNICEF han ido organizando capacitaciones y talleres en colaboración con organizaciones eclesiásticas, ONG locales y municipalidades de la zona fronteriza. Tales talleres y capacitaciones tienen el objetivo de "sensibilizar" a los agentes policiales y profesionales de ONG con respecto a los derechos humanos de migrantes, por un lado, y por otro, en cuanto a "señales" de que una persona podría pertenecer a un grupo de traficantes o tratantes de personas o haber sido víctima de los mismos. Un funcionario de la Policía de Migración de Upala, al ser entrevistado en el marco de la presente investigación, comentó que en una de estas capacitaciones los supervisores de la OIM les habían orientado estar más atentos que antes a personas foráneas o sospechosas que circulan en el espacio fronterizo y, en cambio, tratar con más respeto y no revisar los documentos de la población móvil de las comunidades vecinas nicaragüenses, por ejemplo, los escolares que vienen a Upala o los trabajadores migrantes que laboran en las plantaciones (entrevista a funcionario de la Policía de Migración, Upala, 29 de junio de 2010).

En conjunto, todos los funcionarios de la Fuerza Pública, de la PCD y de la Policía de Migración, así como representantes de organizaciones eclesiásticas y de ONG que se 
entrevistaron, mencionaron el tema de la trata de personas como un problema grave del espacio fronterizo. Ninguno de los informantes, en cambio, pudo nombrar -ni del lado del autor ni del lado de la víctima- un solo caso comprobado.

\section{Zonas de tolerancia y control selectivo de movimientos}

Tanto los actores y las prácticas de movilidad transfronteriza como las de control y management que se han esbozado arriba forman un nuevo régimen de frontera en la zona norte de Costa Rica. Propondría conceptualizar sus elementos centrales como creación de "zonas graduadas de tolerancia" y "criminalización selectiva de prácticas del cruce irregular de frontera".

Estos conceptos apuntan que las fuerzas de seguridad dentro de determinadas zonas, toleran ciertos movimientos y prácticas de cruces de frontera irregulares, y otros no. Tolerancia se muestra, i.e., hacia los escolares de las comunidades del lado nicaragüense o hacia los trabajadores temporales de Nicaragua que, con frecuencia, ingresan irregularmente al lado costarricense con el objetivo de ir a la escuela o ir a trabajar en las plantaciones de productos agrícolas. Ninguno de estos grupos tendrá problema con los funcionarios de la policía siempre y cuando no intenten salir del "espacio fronterizo", definido por las fuerzas de seguridad, hacia el centro de Costa Rica. Individuos móviles, en cambio, que parezcan "foráneos" o "sospechosos" a los funcionarios de la policía, es decir que las fuerzas de seguridad sospechen que son traficantes o tratantes o víctimas de los mismos -i.e. migrantes irregulares de tránsito de África o de Sudamérica-, podrían tener problemas en los puestos de control al intentar ingresar a este espacio fronterizo y pasar por él desde el centro del país. El nuevo delito de "tráfico ilícito de migrantes", además, hace posible criminalizar y penalizar a los transportistas, particularmente a los choferes de taxis piratas siempre y cuando la policía pare y controle a un taxista y éste lleve a personas que no dispongan de los documentos requeridos para ingresar a Costa Rica o egresar a Nicaragua.

Estas formas de control selectivo basadas en tolerancia, criminalización y victimización, recurren al "discurso antitrafficking", anclado en la nueva ley de migración. De esta manera se construye al individuo móvil como un potencial portador de riesgos o como víctima.

\section{6. "Discursos securitarios” y el miedo como modo de subjetivación}

Al nivel subjetivo de la población móvil de los pueblos fronterizos como Upala, que viven en la frontera, con la frontera y de la frontera, los "discursos securitarios" y sus materialidades se articulan como percepciones que a primera vista parecen contradictorias. Por un lado se puede observar un creciente temor colectivo a la militarización, mayor vigilancia policial y criminalización de la propia vida cotidiana, marcada por cruces irregulares de la frontera. Por otro lado, existe un miedo, también colectivo, a una penetración y dominación del espacio fronterizo por redes ajenas del crimen organizado transnacional. En breve: se siente una creciente inseguridad.

Estas sensaciones de inseguridad social y pública, es decir, el miedo de que se puedan criminalizar las propias prácticas cotidianas y de sobrevivencia (i.e. los taxistas temen 
perder su trabajo) por un lado, y el miedo a una amenaza "foránea" (i.e. que los carteles mexicanos o colombianos puedan penetrar la zona e importar sus prácticas violentas) por el otro, constituyen una expresión local de los discursos dominantes globales y regionales sobre migración irregular e (in)seguridad que se han analizado en este ensayo. Y es precisamente este miedo colectivo ambivalente el que forma la base de la nueva gobernabilidad de la frontera norte de Costa Rica.

\section{Notas}

1 Subdirector de la Fuerza Pública (policía nacional), Zona Norte, de Costa Rica .

2 Este texto se elaboró en base a una ponencia con el mismo título realizada el 2 de septiembre de 2010 en el Instituto de Investigaciones Sociales (IIS) de la Universidad de Costa Rica (UCR).

3 A propósito de delimitar movimientos migratorios transfronterizos y de carácter indocumentado, ilegalizado o clandestino del término criminalizante "ilegal", en el marco de este ensayo se asumirá el concepto de "migración irregular" propuesto por la GCIM, definiendo así inmigraciones, emigraciones y migraciones de tránsito que se realicen fuera de las políticas públicas de regulación de la migración. Las mismas varian de país a país y además son objeto de cambiantes coyunturas políticas y legislativas así que el estatus y las prácticas de una persona migrante pueden ser legales o ilegales dependiendo del correspondiente contexto en lo cual se mueve. Con respecto a datos estadísticos resulta difícil, por la naturaleza indocumentada, estimar el número de personas migrantes irregulares en el mundo. Khalid Koser habla de una cantidad de alrededor de 30 millones de migrantes irregulares (Koser, 2005, p. 9).

4 Recurriendo a los "critical security studies" elaborados por las denominadas Escuelas de Copenhague y de París, seguiré una concepción constructivista de "seguridad" (pública). Esto quiere decir que los debates institucionales y públicos sobre el fenómeno de la migración y sus implicaciones para la seguridad pública contribuyen a la construcción de la migración como un problema de seguridad. Las discusiones sobre la migración irregular no son, por ende, simples descripciones de la realidad, sino parte de la construcción de la realidad (véase C.A.S.E. Collective, 2006).

5 Véase i.e. Bigo (2000), Bigo y Guild (2005), Waever, Buzan, Kelstrup \& Lemaitre (eds.) (1993), Waever (1995) y Weiner (1992; 1995) para el caso de Europa; así como i.e. Dunn (1996), Payan (2006), Rochlin (1997), Sandoval (2005) y Specht (2008) en lo que concierne a Norteamérica.

6 Véase http//www.iom.int/jahia/jahia/about- migration/facts-and-figures/lang/en. Recuperado el 03 de noviembre de 2010.

7 No dispongo de datos actualizados principalmente porque el actual Censo de los EE.UU aún no ha terminado.

8 En este caso tampoco dispongo de datos actualizados porque el actual censo costarricense aún no se ha concluido.

9 Véase i.e. Caamaño (2010), Huhn (2005), Jiménez (2009), Morales (2007), Rocha (2004), Sandoval (2007).

10 Habría que mencionar que a principios del año 2010 esta parte del texto se quitó de la entrada de la página web de la OIM en México y Centroamérica (ver http://iom.int/jahia/Jahia/activities/pid/441; 02/04/09).

11 Con Siegfried Jäger (2001) entiendo por "discurso", un conjunto de prácticas sociales interrelacionadas con la percepción y acepción de la realidad social.

12 En esto me basaré en los resultados preliminares de una investigación de campo de carácter cualitativo, micro sociológico y etnográfico que estuve realizando entre marzo y septiembre de 2010 en la capital de Costa Rica, en las regiones fronterizas del país y en Nicaragua.

13 A continuación seguiré usando las siglas del nombre español de la IOM, es decir "Organización Internacional para las Migraciones" (OIM).

14 Entre los RCP más consolidados están la "Conferencia Regional de Migración" (CRM o Proceso Puebla) $\llbracket$ y la "South American Conference on Migration" (SACM) en las Américas; la "Bali Ministerial Conference on People Smuggling, Trafficking in Persons and Related Transnational Crime" (Bali Process) $\square$ y el "Manila Process" en Asia; el "Budapest Process" y el "Cross Border Cooperation Process" (Söderköping Process o CBCP) en Europa; así como el "Mediterranean Transit Migration Dialogue" (MTM), el "Migration Dialogue for West Africa" (MIDWA) y el "Migration Dialogue for Southern Africa" (MIDSA) para el espacio transregional Europa/África (Hansen, 2010).

15 Concluyendo este apartado quisiera agregar que no trato de negar la existencia de carteles criminales de tráfico y trata de personas que operan a nivel global. Lo que quiero dudar es que estas redes tengan una extensión y un impacto tan fuerte que justifique la importancia y prioridad que se le está dando a la lucha contra los mismos en el marco de los RCP.

16 Se trataba i.e. de estandarizar regionalmente los requisitos para obtener visas. 
17 La ley de 2006 entró en vigor pero nunca fue reglamentada por resistencia del gobierno de Oscar Arias (Konrad Adenauer Stiftung, Auslandsbüro Costa Rica 2009. Recuperado el 14 de mayo de 2010 de http://www.kas.de/proj/home/pub/53/1/ dokument_id-16725/index_print.html.

18 En el caso de que se logre comprobar que se trata de un "grupo organizado de dos o más personas" la condena de prisión se puede aumentar incluso hasta ocho años (Ley General de Migración y Extranjería 8764, título 15, articulo 249.).

19 Se cambió el apellido original.

20 Entrevistas a párroco de Liberia, 23 de abril de 2010; párroco de Upala, 27 de abril de 2010; operadores casa del migrante, Los Chiles, 28 de junio de 2010; y subdirector migración de Upala, 29 de junio de 2010.

21 Entrevistas a coordinadora ONG, Upala, 27 de abril de 2010; párroco de Upala, 27 de abril de 2010; taxista de Upala, 27 de abril de 2010.

\section{Referencias bibliográficas}

Bernecker, W. (2005). Staatliche Grenzen. Kontinentale Dynamik. Zur Relativität von Grenzen in Lateinamerika. En M. Braig, O. Ette, D. Ingenschay \& G. Maihold (Eds.). Grenzen der Macht - Macht der Grenzen. Lateinamerika im globalen Kontext. Frankfurt am Main: Verfuert, pp. 11-37.

Bigo, D. \& Guild, E. (Eds.) (2005). Controlling Frontiers. Free Movement Into and Within Europe. Aldershot: Ashgate.

Bigo, D. (2000). Border Regimes and Security in an Enlarged European Community Police Cooperation with CEEs: Between trust and Obligation. EUI Working Papers RSC (65).

Braig, M. \& Baur, C. U. (2005). Mexikos Süden: Grenzüberschreitungen und die Schleusen hemisphärischer Sicherheit. En M. Braig, O. Ette, D. Ingenschay \& G. Maihold (Eds.). Grenzen der Macht - Macht der Grenzen. Lateinamerika im globalen Kontext. Frankfurt am Main: Verfuert, pp. 181-206.

Brand, U. (2000). Global Governance. Alternative zur neoliberalen Globalisierung? Münster: Westfälisches Dampfboot.

C.A.S.E. Collective. (2006). Critical Approaches to Security in Europe. A Network Manifesto. Security Dialogue (37), 443.

Caamaño, C. (2010). Entre 'Arriba' y 'Abajo'. La experiencia transnacional de la migración de costarricenses hacia Estados Unidos. San José: Editorial UCR.

Castillo, M. Á. (2003). The Mexican-Guatemalan Border. New Controls on Transborder Migrations in View of Recent Integrations Schemes?. Frontera Norte, 15 (29), 35-64.

Código Penal de Costa Rica, No. 4573 (2009). Recuperado el 08 de septiembre de 2010 de http://www.aiamp.net/fichasaiamp/costarica/codigo_penal_cr.pdf

Dunn, T. (1996). The Militarization of the U.S.-Mexican Border, 1978-1992. Low Intensity Conflict Doctrine Comes Home. Austin: University of Texas Press.

Foucault, M. (1991). Governmentality. En G. Burchell, C. Gordon, \& P. Miller (Eds.). The Foucault Effect: Studies in Governmentality. Chicago: University of Chicago Press, pp. $87-104$.

Geiger, M. \& Pécond, A. (Eds.) (2010). The Politics of International Migration Management. Basingstike: Palgrave McMillan.

Georgi, F. (2010). International Organization of Migration (IOM). Eine kritische Analyse. En S. Hess \& B. Kasparek (Eds.). Grenzregime. Diskurse. Praktiken. Institutionen in Europa. Berlin: Assoziation A, pp. 145-157.

Girot, P. \& Granados, C. (1997). La cooperación transfronteriza y los nuevos paradigmas para la integración centroamericana. En P. Bovin (Ed.). Las fronteras del Istmo. Fronteras 
y sociedades entre el Sur de México y América Central. México D.F.: CIESAS/CEMCA, pp. 289-310.

Girot, P. (1989). Formación y estructuración de una frontera viva: el caso de la región norte de Costa Rica. Geoistmo, 3 (2), 17-42.

Glick Schiller, N. \& Wimmer, A. (2003). Nationalism, the Social Sciences, and the Study of Migration. An Essay in Historical Epistemology. International Migration Review, 37, (3), 576-610.

Global Commission on International Migration (GCIM). (2005). Migration in the Americas. A paper prepared for the Policy Analysis and Research Programme of the Global Commission on International Migration. Recuperado el 29 de julio de 2010 de www. un.org/esa/population/meetings/fourthcoord2005/P09_GCIM.pdf

Grund, K. (2006). 'Su lucha es nuestra lucha. Juntos ganaremos.' Zentralamerikanische Migranten in den USA und das CAFTA-Abkommen. Ibero-Analysen (17).

Hansen, R. (2010). An Assessment of Principal Regional Consultative Processes on Migration. IOM Migration Research Series No. 38. Ginebra: International Organization for Migration.

Hess, S. \& Kasparek, B. (Eds.) (2010). Grenzregime. Diskurse. Praktiken. Institutionen in Europa. Berlin: Assoziation A.

Hess, S. \& Tsianos, V. (2007). Europeanizing Transnationalism! Provincializing Europe! Konturen eines neuen Grenzregimes. En Transitmigration Forschungsgruppe (Ed.) Turbulente Ränder. Neue Perspektiven auf Migration in Europa. Bielefeld: transcript, pp. 23-38.

Huhn, S. (2005). Einwanderungsdiskurse und Migrationspolitik in Costa Rica. Wandel und Konstanten in der gesellschaftlichen Auseinandersetzung mit der nikaraguanischen Migration seit den 1980er Jahren. (Beiträge zur Lateinamerikaforschung 18). Hamburg: Institut für Iberoamerika-Kunde

International Organisation for Migration (IOM). (2002). Workshop on Migration Management and Improving Inter-State Cooperation held under the auspices of the Regional Conference on Migration in Guatemala City, on 28 October 2002. IOM Migration and Policy Research Programme (MPRP). Recuperado el 29 de julio de 2010 de www.rcmvs.org/Eventos/Guatemala_Workshop_Final_Report.doc

International Organisation for Migration (IOM). (2002b): The State of Migration Management in Central America: An Applied Research Final Report. Recuperado el 29 de julio de 2010 de www.acnur.org/t3/fileadmin/Documentos/nuevo_sitio/2010/ conferencia/4.\%20Mixed\%20Migration $\% 20$ Flows\%20in\%20the\%20Americas/IOM\%20 -\%20The \%20State\%20of\%20Migration\%20Management\%20in\%20Central\%20America. pdf

International Organisation on Migration (IOM). (1998). Trafficking in Migrants: Some Global and Regional Perspectives. Paper presented at the Seminar on Migrant Trafficking as a Follow-up of the Puebla Process, 22-23. Managua.

Jäger, S. (2001). Kritische Diskursanalyse. Eine Einführung. Duisburg: DISS.

Jiménez, A. (2009). La vida en otra parte. Migraciones y cambios culturales en Costa Rica. San José: Editorial Arlekín.

Karakayali, S. \& Tsianos, V. (2007). Movements that Matter. Eine Einleitung. En: Transit Migration Forschungsgruppe (Hg.): Turbulente Ränder. Neue Perspektiven auf Migration an den Grenzen Europas. Bielefeld: transcript, pp. 7-17. 
Keohane, R. O. (1982). The Demand for International Regimes. International Organization, $36(2), 325-355$

Keohane, R. O. (1984). After Hegemony. Cooperation and Discord in the World Political Order. Princeton: Princeton University Press.

Klein-Solomon, M. (2005). International Migration Management through Inter-State Consultation Mechanisms. Focus on Regional Consultative Processes on Migration, IOM's International Dialogue on Migration and the Berne Initiative. Recuperado el 03 de noviembre de $2010 \mathrm{de}$ http//www.un.org/esa/population/meetings/ittmigdev2005/ P13_MKSolomon.pdf

Klekowski von Koppenfels, A. (2001). The Role of Regional Consultative Processes in Managing International Migration. IOM Migration Research Series No. 3. Ginebra: International Organization for Migration.

Koser, K. (2005). Irregular migration, State security and Human security. A paper prepared for the Policy Analysis and Research Programme of the Global Commission on International Migration. Recuperado el 03 de noviembre de $2010 \mathrm{de} \mathrm{http://www.}$ gcim.org/attachements/TP5.pdf

Ley General de Migración y Extranjería de Costa Rica No. 7033 (1986).

Ley General de Migración y Extranjería de Costa Rica No. 8764 (2010).

Medina-Nicolas, L. (2004). Le Dilemme des Frontières en Amérique Centrale: Marges Symboliques ou Espaces en Construction? Le Cas des Frontières Nicaragua-Costa Rica et Costa Rica-Panama. (Tesis de doctorado no publicada, Paris).

Morales Gamboa, A. (2007). La diáspora de la posguerra. Regionalismo de los migrantes y dinámicas territoriales en América Central. San José: FLACSO.

Núñez, R. \& Marín, J. J. (Eds.) (2009). Guanacaste: Historia de la (Re)Construcción de una región. 1850-2007. San José: Editorial UCR.

Papadopoulos, D., Stephenson, N. \& Tsianos, V. (2008). Escape Routes. Control and Subversion in the 21st Century. London: Pluto Press.

Payan, T. (2006). The Three U.S.-Mexico Border Wars: Drugs, Immigration and Homeland Security. Westport, CT: Praeger.

Regional Conference on Migration (RCM). (2004). The Regional Conference on Migration (RCM). In brief: what it is, what it has done. Meeting of the Regional Consultation Group on Migration (RCGM). May, 18-19, 2004, Panama City. Recuperado el 29 de julio de 2010 de www.rcmvs.org/crm_en_breve_v13-05-04-eng.doc

Regional Conference on Migration (RCM). (2006). Experience of the Technical Secretariat of the Regional Conference on Migration. Workshop on Regional Consultative Processes, United Nations Headquarters, June 20, 2006, New York. Recuperado el 29 de Julio de 2010 de www.globalmigrationgroup.org/pdf/UNITARFinalRCPsSummaryRepor t_20June2006.pdf

Robinson, W. (2003). Transnational Conflicts. Central America, Social Change, and Globalization. London/New York: Verso.

Rocha, J. L. (2004). Migración internacional en Centroamérica. Mapeo regional de flujos, leyes, organismos e investigaciones en torno a los migrantes centroamericanos. Working Papers Series CST/LAC (37), diciembre. Managua: UNFPA-Equipo de Apoyo Técnico para América Latina y el Caribe.

Rocha, J. L. (2006). A Region Torn Apart. The Dynamics of Migration in Central America/Una región desgarrada. Dinámicas migratorias en Centroamérica. 
Managua: Servicio Jesuita para Migrantes / Red Nicaragüense de la Sociedad Civil para las migraciones.

Rochlin, F. L. (1997). Redefining Mexican "Security". Society, State and Region under NAFTA. London: Lynne Rienner Publishers.

Sandoval, C. (Ed.) (2007). El mito roto. Inmigración y emigración en Costa Rica. San José: Editorial UCR.

Sandoval, J. M. (2005). México: País Frontera. La nueva frontera geopolítica regional de América del Norte. En J. M. Sandoval \& y R. Álvarez (Eds.). Integración Latinoamericana, Fronteras y Migración: los casos de México y Venezuela. México D.F.: Centro de estudios chicanos y de fronteras/Plaza y Valdés, pp. 65-100.

Specht, J. (2008). Securitización de la migración y derechos humanos en EE.UU y México: ¿amenaza u oportunidad para una ampliación de la ciudadanía?. En B. Potthast, J. Ströbele-Gregor \& D. Wollrad (Eds.). Ciudadanía vivida, (in)seguridades $e$ interculturalidad. Buenos Aires: Friedrich Ebert Stiftung/Adlaf/Nueva Sociedad, pp. 300-317.

Thouez, C. \& Channac, F. (2005). Regional Consultative Processes for Migration: An Evaluation Based on IMP's Work. New York: UNFPA.

Transit Migration Forschungsgruppe (Ed.)(2007). Turbulente Ränder. Neue Perspektiven auf Migration an den Grenzen Europas. Bielefeld: transcript.

UN Convention Transnacional Crime, Annex III. (2000 [2004]). Protocol against the Smuggling of Migrants by Land, Sea and Air, supplementing the United Nations Convention against Transnational Organized Crime.

UN Convention Transnational Crime, Annex II. (2000 [2003]). Protocol to Prevent, Suppress and Punish Trafficking in Persons Especially Women and Children, supplementing the United Nations Convention against Transnational Organized Crime.

US Census. (2002). Statistical Abstracts of the United States 2001. The National Data Handbook. Hoover Business Press.

Villa, M. \& Pizarro, J. (2002). Trends and Patterns of International Migration in Latin America and the Caribbean. Paper prepared for the Simposio sobre migración internacional en las Américas. Sponsored by the United Nations, CEPAL, and ECLAC. San José, Costa Rica.

Villalobos, L. B., Chamizo, H. \& Piedra, M. A. (2008). Cuando la Vulnerablilidad se desplaza. VIH/Sida y poblaciones móviles en la estación de paso La Cruz, Costa Rica. San José: Editorial UCR.

Waever, O. (1995). Securitization and Desecuritization. En R. Lipschutze (Ed.). On Security. New York: Columbia University Press, pp. 46-86.

Waever, O., Buzan, B., Kelstrup, M. \& Lemaitre, P. (Eds.) (1993). Identity, Migration and the New Security Agenda in Europe. London: Pinter.

Weiner, M. (1992). The Global Migration Crisis: Challenge to State and Human Rights. New York: Addison-Wesley Longman Publishers.

Weiner, M. (1995). Security, Stability and International Migration. En International Security, 17 (3), 91-126.

World Bank. (2008). Migration and Remittanes Factbook 2008. Washington: World Bank. 